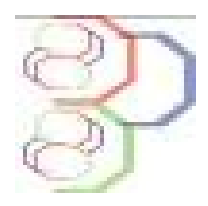

Journal of Applied Biosciences 61: $4515-4529$

\title{
L'arbre en milieu soudano-sahélien dans le bassin arachidier (Centre-Sénégal)
}

\author{
Oumar Sarr ${ }^{*}$, Amy Bakhoum ${ }^{1}$, Sékouna Diatta ${ }^{1}$, Léonard .E. Akpo ${ }^{1}$ \\ ${ }^{1}$ UCAD/FST Laboratoire d'Ecologie et d'Ecohydrologie, BP n 5005 Dakar - Fann, SENEGAL \\ *Correspondance : Oumar SARR, Email : oumarsarr2@yahoo.fr \\ Original submitted in on 13th December 2012. Published online at www.m.elewa.org on 31st January 2013.
}

\begin{abstract}
RESUME
Objectifs : L'objectif est de déterminer le rôle de l'arbre et sa place dans les parcours communautaires de Kaffrine.

Méthodologie et résultats : des questionnaires appliqués aux transhumants et aux résidents des villages retenus pour les deux sites. Leur contenu a abordé des sujets liés à la perception du rôle de l'arbre. Les résultats indiquent globalement un niveau très élevé de consensus (supérieur à $60 \%$ pour l'ensemble des usages). Cela prouve que l'arbre occupe une place très importante dans la vie des ruraux. Dans la zone, l'arbre est tout d'abord utilisé pour l'alimentation du bétail, dans la pharmacopée, puis dans l'alimentation humaine...L'AFC indique un lien espèces-usages très fort avec des espèces à usages multiples témoignant d'une forte pression sur la ressource.

Conclusion et application : Le présent travail montre la place et la fonction que l'arbre occupe dans le vécu quotidien des agriculteurs et pasteurs dans les parcours des communautés rurales de Lour Escale et de Ida Mouride. Les ligneux assurent des fonctions aussi importantes et diverses et jouent ainsi un rôle social très important. Ils interviennent dans : l'alimentation du bétail et des humains, la pharmacopée, l'artisanat, la production de bois d'énergie et de charbon etc. Dès lors, des moyens de gestion et de réhabilitation des écosystèmes doivent être analysés avec les populations afin de permettre aux décideurs publics et privés d'avoir des outils pratiques pour une exploitation rationnelle de la ressource dans les parcours communautaires afin d'assurer leur durabilité.
\end{abstract}

Mots clés : perception, arbre, usages, AFC, écosystème, réhabilitation

The Role of Trees in the soudano-sahelian zone of the groundnut basin in Senegal (Western Africa) ABSTRACT

Objectives: To determine the role of trees in the pasture land of Kaffrine communities.

Methodology and results: Ethnobotanical surveys applied to transhumant and local population were carried out in the selected villages in the two sites. Content addressed topics related to the perception on the role of trees. Results indicate a very high level of consensus (greater than $60 \%$ for all uses). This proves that woody species occupies a very important place in the lives of rural people. In this area trees are first used for livestock feed, in pharmacopoeia, and in food for men... The FCA indicates a high species-purpose link with multipurpose species showing a strong pressure on the resource.

Conclusion: The present work shows the role and function that the trees play in the daily lives of farmers and pastoralists in the pasture lands of Lour Escale and Ida Mouride rural communities. Wood diverse 
and multiple functions and thus plays a very important social role. These roles includes: Feeding cattle and humans, medicine, crafts and production of firewood.

Therefore, the means of management and rehabilitation of ecosystems should be analyzed with people to enable public and private decision to have practical tools for rational exploitation of the resource in the community.

Key words: perception, tree, use, FCA, ecosystem, rehabilitation

\section{INTRODUCTION :}

Les agriculteurs et les pasteurs, ainsi que l'ensemble de la population rurale, ont des connaissances traditionnelles sur les arbres, du fait de leur utilisation ancestrale. Dans les terroirs villageois, l'arbre, joue plusieurs rôles dans les systèmes de production et occupe une place très importante dans la vie des populations. II permet le développement de la strate herbacée, améliore la fertilité du sol (Akpo et Grouzis, 1996 ; Akpo, 1997). En milieu pastoral, l'arbre fournit en saison sèche au bétail les compléments en protéines et en vitamines indispensables à sa survie (Le Houerou, 1980 ; Breman et al, 1991). II peut aussi servir à la fois dans l'alimentation des Hommes et

\section{MATERIEL ET METHODES}

La zone d'étude : L'étude a été réalisée dans le centre du Sénégal, dans la région administrative de Kaffrine située entre les latitudes $12^{\circ} 06 \mathrm{~N}$ et les longitudes $15^{\circ} 330$. Elle est limitée au Nord par les régions de Diourbel, Louga et Matam, au Sud par la République de Gambie, à l'Est par la région de Tambacounda et à l'Ouest par la région de Kaolack (figure 1). L'étude a été réalisée spécifiquement dans deux communautés rurales de la région de Kaffrine, Lour Escale et Ida Mouride. En effet, il s'agit d'une zone de transition écologique entre le Nord sahélien à vocation pastorale et le Sud Soudanien, qui accueille ou dans laquelle transitent de nombreux ruminants dans la saison sèche, et dont l'avancée du front agricole exerce une forte pression sur les ressources ligneuses disponibles. Le climat tropical sec est de type soudano-sahélien. Les températures mensuelles moyennes minimales et maximales sont respectivement de $16,2^{\circ} \mathrm{C}$ (janvier) et $42,1^{\circ} \mathrm{C}$ (mai). La température moyenne annuelle est de l'ordre de $29,6^{\circ} \mathrm{C}$. Les précipitations sont irrégulières: la pluviométrie moyenne (entre 1965 et 2008) est de $704,32 \mathrm{~mm}$ à la station de référence (Koungheul). des animaux (Boudet, 1975 ; Giffard, 1974; Von Maeydell, 1990), être utilisé pour l'énergie domestique, dans la construction d'habitats ou de clôture des maisons et des jardins, dans la pharmacopée traditionnelle comme moderne. Malheureusement, les effets cumulés des sécheresses récurrentes, de certaines pratiques liées à l'agriculture rendent vulnérables des espèces (Betti, 2007) si bien que certaines en arrivent même à disparaître (Sarr, 2009). La présente étude vise à appréhender le rôle de l'arbre et sa place dans la vie des populations principalement dans les parcours communautaires de Kaffrine.

Koungheul est caractérisé par un déficit pluviométrique persistant depuis 1970, avec une grande proportion d'années biologiquement sèches, conduisant à une quantité totale de pluie recueillie inférieure à la moyenne annuelle correspondant à 23 années de déficit (figure 2). La saison des pluies survient de mai à octobre, ce qui permet de distinguer classiquement deux périodes dans l'année: une période sèche $(P<$ 2T) de 7 à 8 mois (d'octobre à mai) et une saison des pluies $(P>2 T)$ de 4 à 5 mois (figure 3 ). Les mois de juillet, août et septembre totalisent $79,31 \%$ des précipitations, constituent la période biologiquement humide, le mois d'août étant le plus pluvieux (32\%) (Figure 3). La région de Kaffrine appartient aux formations sédimentaires du continental terminal (Touré, 2002). Les sols sont sableux, sablo-argileux ou argilo-sableux et supportent une végétation caractérisée du Nord au Sud par une savane herbeuse, une savane arbustive, une savane arborée et une savane boisée. L'agriculture, essentiellement sous pluie, et l'élevage extensif constituent les principales activités de la région. 


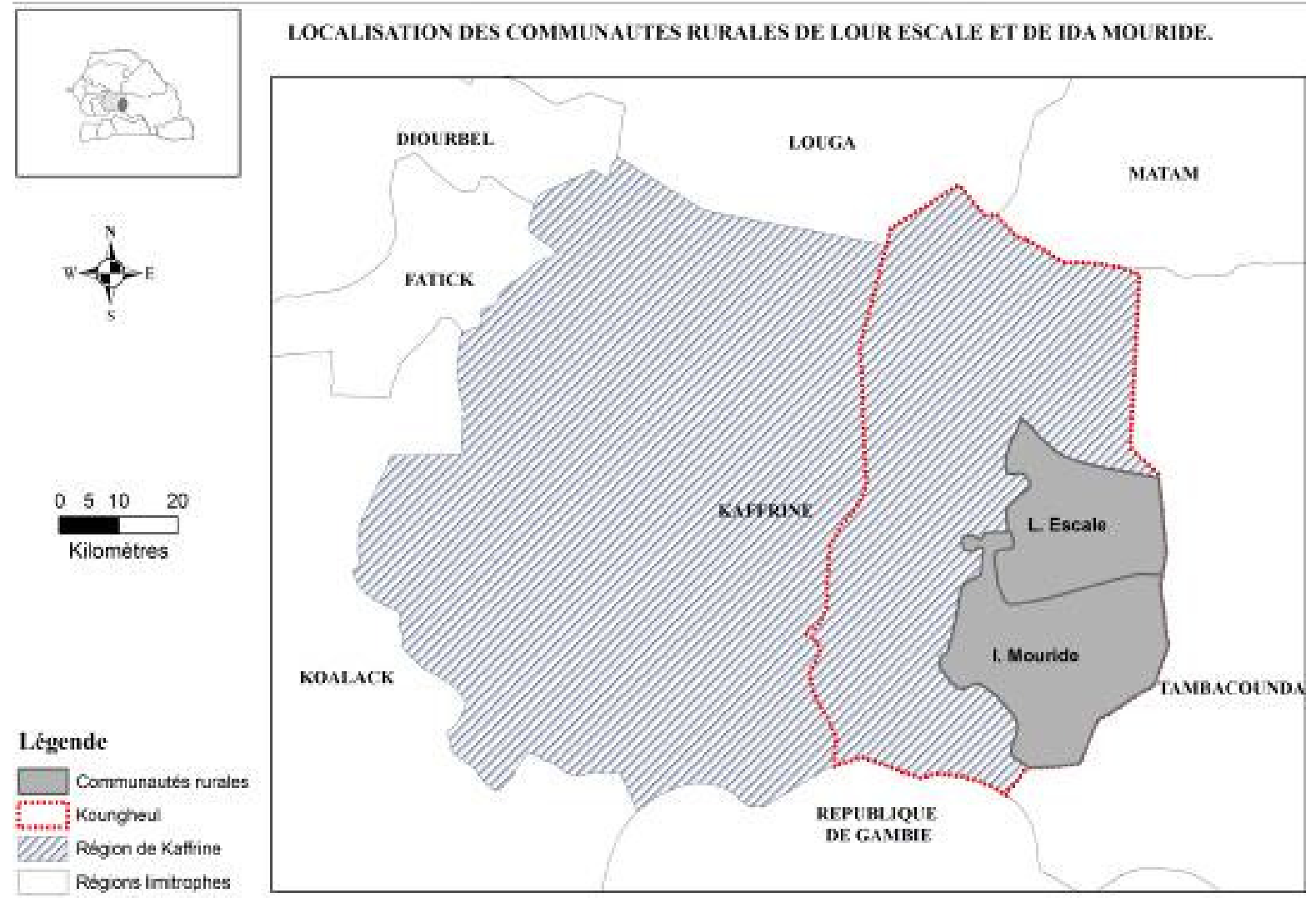

Figure 1 : Situation géographique de la zone d'étude (communautés rurales de Lour Escale et de Ida Mouride).

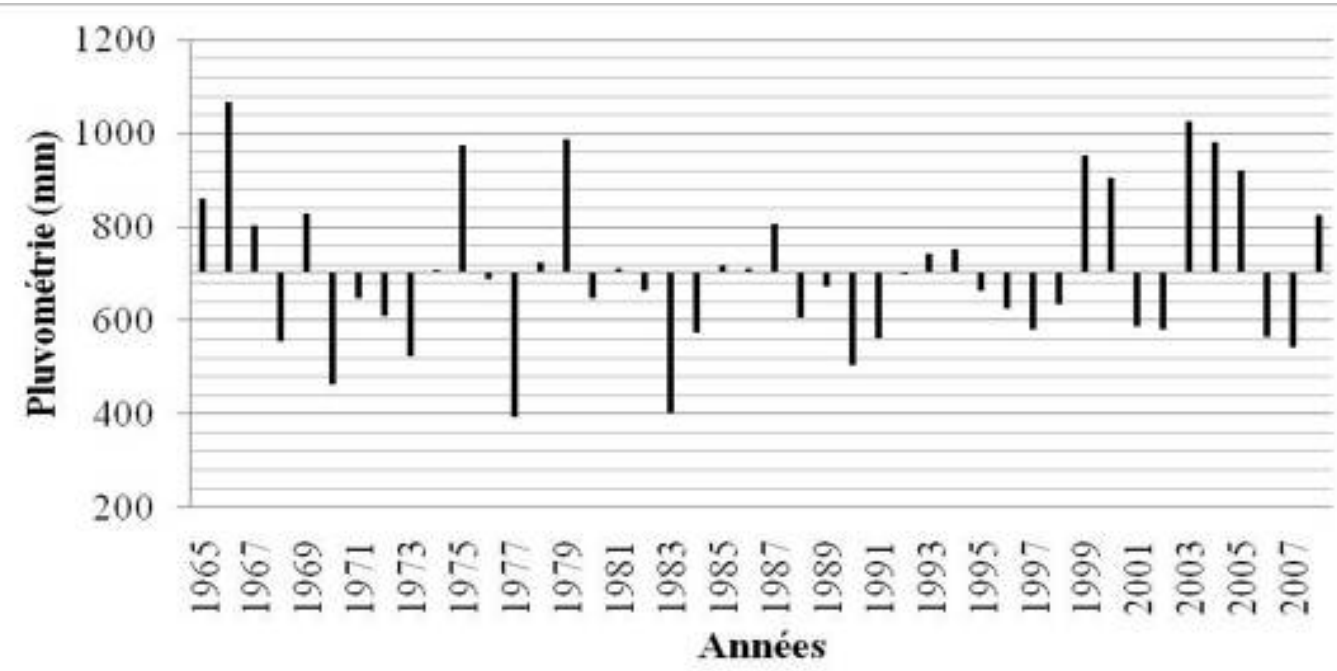

Figure 2 : Variabilité interannuelle de la pluviométrie de la station de Koungheul (de 1965 à 2008) (source ANAMS). 


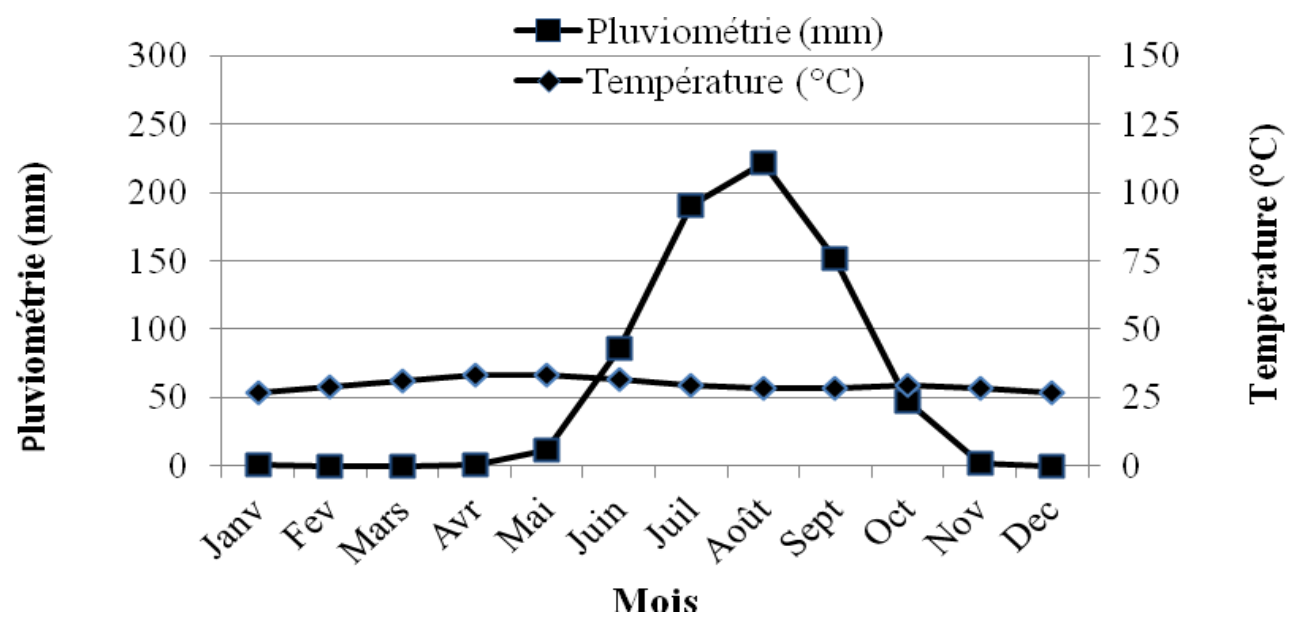

Figure 3 : Diagramme ombrothermique de la communauté rurale de Lour Escale (source ANAMS). $P$ : pluviométrie moyenne mensuelle de la région de Kaffrine de 2000 à 2008.

$\mathrm{T}$ : température moyenne mensuelle (minimale et maximale) de la région de Kaffrine de 2000 à 2008.

Méthodes de collecte des données : L'enquête a été réalisée au courant des mois d'Avril-Mai 2011 à l'aide de questionnaires appliqués aux transhumants (15 fiches) et aux résidents des villages retenus (30 fiches) pour les deux sites de l'étude (Communautés rurales de Lour Esacale et d'lda Mouride). Cette période correspond au moment où les transhumants (ceux qui y sont installés et ceux qui reviennent des zones plus au Sud) s'y retrouvent avant de remonter vers le Nord et où l'herbe est quasi inexistante. Nous avons aussi réalisé, au niveau de chaque terroir villageois, des interviews auprès des acteurs concernés, en faisant des focus group (FAO, 1992). Le choix des informateurs est fait avec l'aide du chef de village en tenant compte de l'âge. Ont été enquêtés, des individus dont les âges varient entre 40 et 75 ans ayant une bonne connaissance du milieu et des ressources ligneuses locales. Le contenu des questionnaires a abordé des sujets liés aux activités des populations (exploitation des ressources, outillage agricole et spéculations), à la transhumance (historique, organisation, axes de déplacement, etc.) et aux espèces ligneuses rencontrées (importance, usages, rôles, état actuel, dynamique). Dans ce présent travail, nous traiterons uniquement les données relatives à la perception du rôle de l'arbre dans les paysages villageois. Les techniques d'exploitation et de conservation utilisés seront aussi inventoriées et analysées avec les populations afin d'identifier les préférences et les usages, les contraintes liées à la gestion durable des espèces et l'importance des ligneux fourragers dans ces parcours.

Traitement des données : Les données des enquêtes ont été d'abord dépouillées manuellement puis saisies et traitées dans le logiciel Sphinx Plus et le tableur Excel. Sphinx est un logiciel d'enquête qui génère directement des résultats à partir des données saisies et permet des analyses comparatives entre deux séries de questions ou entre deux tableaux de valeurs. La dénomination des espèces a été effectuée sur la base de la Flore du Sénégal (BERHAUT, 1967), de l'ouvrage des "noms vernaculaires des plantes » (ADAM, 1970) et du catalogue des plantes vasculaires du Sénégal (LEBRUN et STORK, 1991 ; 1992 ; 1995 et 1997).

La Valeur Usuel (VU) des espèces pour chaque catégorie d'usage est évaluée pour montrer l'importance que les personnes accordent à une espèce donnée dans la localité. Elle est obtenue en calculant le rapport suivant :

$\mathrm{VU}=\Sigma \mathrm{U} / \mathrm{N}$

Où $U$ représente le nombre de fois qu'une espèce est citée pour une catégorie d'usage et $\mathbf{N}$ le nombre total d'informateurs.

Nous avons utilisé le Facteur de Consensus Informateur (FIC) que nous avons réadapté pour caractériser les essences dans six (6) catégories d'usages : pâturage ; aliment ; médecine ; artisanat ; bois de feu et bois de service. II sert à estimer la variabilité des utilisations des espèces. Sa valeur varie entre 0 et 1. 
Il est obtenu en utilisant la formule suivante (Canales et al, 2005):

FIC $=$ Nur - Nt $I($ Nur -1$)$

Avec Nur est le nombre de fois que l'espèce est citée pour une catégorie particulière d'usage et $\mathrm{Nt}$ est le nombre total d'espèces utilisées par tous les informateurs pour cette même catégorie.

Le Niveau de fidélité (FL) (Cheikhyoussef et al, 2011) dégage l'importance que les populations accordent à une espèce pour son rôle. II est calculé par la formule suivante :

\section{RESULTATS}

Les principaux rôles des ligneux, niveau de consensus informateur : Les ligneux rencontrés dans les deux territoires étudiés procurent plusieurs biens et services aux communautés locales. Ces services sont

\section{FL $(\%)=N p / N \times 100$}

Où $\mathrm{Np}$ représente le nombre de citation d'une espèce pour son rôle et $\mathbf{N}$ le nombre totale d'utilisations à toutes fins.

Nous avons soumis une matrice de 47 espèces $\times 6$ types d'usages à une analyse factorielle des correspondances (AFC) en utilisant le logiciel XLSTAT pour identifier d'éventuels groupes espèces-services écosystémiques c'est-à-dire les services que l'arbre offre en temps qu' élément de l'écosystème.

l'alimentation pour le bétail et pour les Hommes, la pharmacopée et l'artisanat entre autres. La figure 4 montre la proportion des différents usages en termes spécifiques.

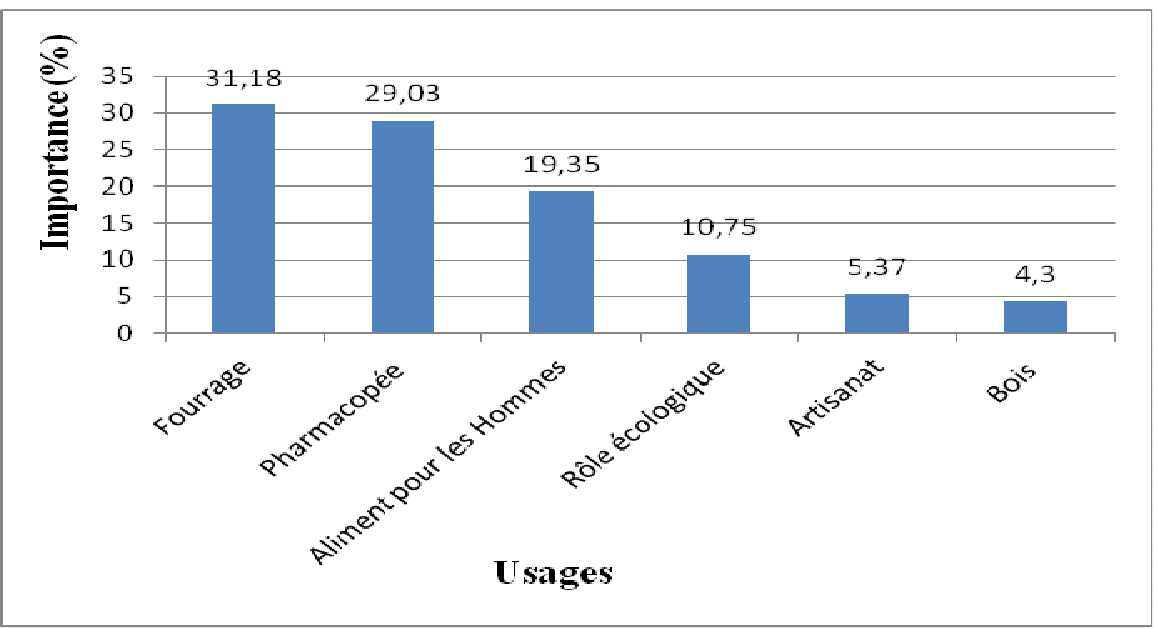

Figure 4: Principaux usages des plantes.

Environ $80 \%$ des espèces sont utilisées pour le fourrage, la pharmacopée et l'alimentation humaine. Les populations utilisent prioritairement l'arbre pour nourrir leur bétail avec un total de 29 espèces (soit $31 \%$ ), pour se soigner ensuite en utilisant 26 espèces ligneuses (soit 29\%) et pour se nourrir avec 18 espèces $(19 \%)$. Suivent ensuite les usages pour le rôle écologique, l'artisanat et pour le bois généralement. II apparait aussi un niveau élevé de consensus pour les différents usages (tableau 1).

Tableau 1 : FIC et VU pour chaque catégorie d'usage.

\begin{tabular}{lcccc}
\hline Usages & N esp & N cit & FIC & VU \\
\hline Pâturage & 29 & 399 & 0,92 & 0,64 \\
Aliment & 18 & 302 & 0,94 & 0,40 \\
Bois de feu & 5 & 37 & 0,88 & 0,11 \\
Médecine & 26 & 237 & 0,89 & 0,57 \\
Artisanat & 7 & 17 & 0,62 & 0,15 \\
Bois de service & 8 & 32 & 0,77 & 0,17 \\
\hline
\end{tabular}

N.esp : nombre d'espèces ; Ncit : nombre de citation ; FIC : Facteur de consensus Informateur ; VU : Valeur Usuelle. 
Le consensus est globalement supérieur à $75 \%$ à l'exception de la valeur pour l'artisanat (62\%). L'arbre pour l'alimentation des hommes et du bétail occupe une place prépondérante dans la vie des populations $\mathrm{du}$ fait d'un niveau de consensus très important (respectivement de 0,94 et 0,92) et avec une valeur usuelle de 0,40 et 0,64 respectivement. Cela montre que dans la localité, les ligneux sont à usages multiples et satisfont unanimement les besoins des populations et du bétail.

Rôle écologique de l'arbre: L'arbre joue un rôle important dans l'amélioration du niveau trophique du sol et dans sa protection. En effet, les populations admettent le rôle de l'arbre dans la stabilisation des dunes de sable. $100 \%$ de la population locale enquêtée l'ont affirmé et aussi la plupart des transhumants dans la zone. Des espèces comme Guiera senegalensis et Faidherbia albida sont particulièrement citées comme participant véritablement à la remontée de la fertilité des sols. Faidherbia albida est totalement épargnée par les coupes du fait de la reconnaissance de son importance dans l'amélioration du rendement agricole. Mais, en ce qui concerne la Guiera senegalensis, elle est reconnue comme étant un bon indicateur de sols sableux, propice à l'agriculture de l'arachide mais elle est systématiquement éliminée dans les terres agricoles. De même, il est reconnu que les ligneux constituent un élément essentiel dans le maintien et la préservation des mares et des puits en réduisant leur ensablement surtout favorisé par les vents.

Rôle dans l'alimentation humaine : Les produits de consommation issus des ligneux constituent une très grande part des prélèvements. Ainsi, 18 espèces ont été citées comme utilisées par les humains comme apport alimentaire. Les feuilles constituent une part très importante dans l'apport alimentaire des populations. Elles sont souvent réduites à l'état de poudre ou préparées en sauce pour accompagner le couscous (Adansonia digitata). Les fruits sont le plus souvent utilisés pour l'autoconsommation ou le petit commerce ; ils proviennent de ligneux comme (Adansonia digitata, Cordyla pinnata, Ziziphus mauritiana, et même parfois Strychnos spinosa). En particulier, le fruit de Cordyla pinnata constitue un véritable apport alimentaire et est très prisé aussi bien par les populations autochtones que les transhumants dans la localité car pouvant remplacer la viande dans la préparation du couscous. Cette espèce constitue d'ailleurs la première en terme de valeur usuelle ( $\mathrm{VU}=1)$, suivie par Adansonia digitata et Gardenia erubescens ( $\mathrm{VU}=0,77$ et $\mathrm{VU}=0,57$ ).

La gomme de Sterculia setigera $(\mathrm{VU}=0,42)$ est particulièrement recherchée pour aussi accompagner le couscous, base de la nourriture du soir et du petit matin. Le tableau 2 indique les espèces ligneuses à usage alimentaire indiquées dans les deux communautés rurales étudiées.

Tableau 2 : Espèces à usage alimentaire, leur valeur usuelle et les parties utilisées.

\begin{tabular}{lccc}
\hline Espèces & Nb. cit. & VU & Parties-utilisées \\
\hline Cordyla pinnata & 45 & 1,00 & Fruits \\
Adansonia digitata & 35 & 0,77 & Feuilles/Fruits \\
Gardenia erubescens & 26 & 0,57 & Feuilles/Fruits \\
Sterculia setigera & 19 & 0,42 & Gomme \\
Sclerocarya birrea & 18 & 0,40 & Fruits \\
Ziziphus mauritiana & 17 & 0,37 & Fruits \\
Combretum micranthum & 16 & 0,35 & Feuilles \\
Moringa oleifera & 15 & 0,33 & Feuilles \\
Balanites aegyptiaca & 14 & 0,31 & Fruits/Huile \\
Anacardium occidentalis & 14 & 0,31 & Fruits \\
Afzelia africana & 13 & 0,28 & Fruits \\
Ziziphus gola & 13 & 0,28 & Fruits \\
Leptadenia hastata & 12 & 0,26 & Feuilles \\
Strychnos spinosa & 11 & 0,24 & Fruits \\
Ficus gnaphalocarpa & 11 & 0,24 & Fruits \\
Terminalia avicenniodes & 9 & 0,20 & Racines \\
Feretia apodanthera & 7 & 0,15 & Feuilles \\
Hannoa undulata & 7 & 0,15 & Fruits \\
\hline
\end{tabular}

$\mathrm{Nb}$ cit : Nombre de citation ; VU : Valeur Usuelle 
Les deux espèces les plus consommées par les populations locales sont Cordyla pinnata et Adansonia digitata. Elles apparaissent comme étant les espèces à usage alimentaire les plus importantes. Concernant les parties utilisées de l'arbre, l'examen du tableau montre que ce sont les fruits qui sont les plus consommés suivis des feuilles et de la gomme ou de l'huile. C'est ainsi que l'huile de Balanites aegyptiaca est très recherchée tout comme la gomme "Mbepp» de Sterculia setigera dans la localité.

Rôle fourrager; Les ligneux fourragers sont le principal recours du bétail en période sèche. L'alimentation des animaux est basée essentiellement sur les pâturages naturels des ligneux, des parcours sans limites en période d'après récolte. Les arbres et les arbustes participent à l'entretien aussi bien des animaux domestiques. Les feuilles sont les parties les plus consommées des ligneux. Elles peuvent être utilisées vertes ou sèches en fonction de la période de l'année. Les fleurs et les fruits de certaines espèces sont aussi utilisés dans l'alimentation du bétail. Les ligneux fourragers les plus appétés des territoires étudiés sont Pterocarpus erinaceus (valeur usuelle de $0,86)$, suivie de Sterculia setigera $(\mathrm{VU}=0,64)$ puis de Guiera senegalensis et Acacia senegal (VU=0,46). II faut cependant noter que dans le cas de Combretum glutinosum, les feuilles sont plus utilisées à l'état de folioles qu'à l'état matures. Le tableau suivant montre les espèces à usage fourrager, leur valeur usuelle, les parties utilisées et leurs consommateurs potentiels.

Tableau 3 : ligneux fourragers et les parties consommées par le bétail.

\begin{tabular}{|c|c|c|c|c|}
\hline Fourrage & Nb. cit. & VU & $\mathrm{Pu}$ & Consommateurs \\
\hline Pterocarpus erinaceus & 39 & 0,86 & Feui & Bo-Ov-Cap \\
\hline Sterculia setigera & 29 & 0,64 & Feui & Bo-Ov-Cap \\
\hline Guiera senegalensis & 21 & 0,46 & Feui & Ov-Cap \\
\hline Acacia senegal & 21 & 0,46 & Feui, Frts & Bo-Ov-Cap \\
\hline Adansonia digitata & 18 & 0,40 & Feui/Frts & Bo-Ov-Cap \\
\hline Cordyla pinnata & 18 & 0,40 & Feui & Bo-Ov-Cap \\
\hline Faidherbia albida & 18 & 0,40 & Feui/Frts & Bo-Ov-Cap \\
\hline Ziziphus mauritiana & 16 & 0,35 & Feui/Frts & Bo-Ov-Cap \\
\hline Anogessus leiocarpus & 16 & 0,35 & Feui & Bo-Ov-Cap \\
\hline Calotropis procera & 15 & 0,33 & Feui & Bo-Ov-Cap \\
\hline Grewia flavescens & 15 & 0,33 & Feui, Frts & Bo-Ov-Cap \\
\hline Bombax costatum & 14 & 0,31 & Feui & Bo-Ov-Cap \\
\hline Leptadenia hastata & 13 & 0,28 & Feui & Bo-Ov-Cap \\
\hline Sclerocarya birrea & 13 & 0,28 & Feui, Frts & Bo-Ov-Cap \\
\hline Combretum micranthum & 13 & 0,28 & Folioles & Ov-Cap \\
\hline Azadirachta indica & 12 & 0,26 & Feui & Ov-Cap \\
\hline Feretia apodanthera & 12 & 0,26 & Feui & Bo-Ov-Cap \\
\hline Combretum glutinosum & 11 & 0,24 & Folioles & Bo-Ov-Cap \\
\hline Boscia angustifolia & 10 & 0,22 & Feui & Bo-Ov-Cap \\
\hline Piliostigma reticulatum & 8 & 0,17 & Feui/Frts & Bo-Ov-Cap \\
\hline Diospyros mespiliformis & 8 & 0,17 & Frts & Bo-Ov-Cap \\
\hline Tamarindus indica & 8 & 0,17 & Feui & Bo-Ov-Cap \\
\hline Mitragyna inermis & 8 & 0,17 & Feui & Ov-Cap \\
\hline Pterocarpus lucens & 8 & 0,17 & Feui & Bo-Ov-Cap \\
\hline Cadaba farinosa & 8 & 0,17 & Feui & Bo-Ov-Cap \\
\hline Khaya senegalensis & 8 & 0,17 & Folioles & Ov-Cap \\
\hline Grewia tenax & 8 & 0,17 & Feui, Frts & Bo-Ov-Cap \\
\hline Grewia bicolor & 7 & 0,15 & Feui/Frts & Ov-Cap \\
\hline Acacia nilotica & 4 & 0,08 & Feui/Frts & Bo-Ov-Cap \\
\hline
\end{tabular}

$\mathrm{Nb}$. Cit : Nombre de citation ; VU : Valeur Usuelle ; Pu : Partie utilisée ; Feui : Feuille ; Frts : Fruits ; Bo : Bovin ; Ov : Ovin ; Cap : Caprin. 
L'examen du tableau montre trois espèces sont particulièrement citées comme étant mieux appréciées par les petits ruminants du fait de leur importante citation par les populations. II s'agit de Guiera senegalensis, Azadirachta indica et Grewia bicolor. Cependant, Azadirachta indica (VU=0,26) est souvent considéré comme un fourrage de rescousse ou de complément. II n'est surtout utilisé que par moment, soit dans le cas où la saison sèche est lointaine et que le fourrage herbacé comme la plupart du fourrage aérien devient rare, soit quand les animaux sont déjà rassasiés et cherchent certainement à combler un désir. Ces ligneux entretiennent ainsi la quasi-totalité des troupeaux, surtout des petits ruminants (tableau 3) grâce à leurs feuillages, leurs fruits et leurs fleurs particulièrement en saison sèche.

La contribution des espèces au rôle fourrager est analysée dans le tableau 4 par étude du niveau de fidélité des espèces par rapports aux différents usages. Nous avons $82 \%$ des essences fourragères avec un niveau de fidélité supérieur ou égal à $50 \%$, ce qui montre que le rôle fourrager de l'arbre est considérable. Nous pouvons scinder le tableau en groupes vue l'importance que les populations accordent à chacune d'entre elles dans la zone :
- Le groupe I: constitué d'espèces avec un niveau maximal de fidélité (100\%). II regroupe des espèces comme Pterocarpus erinaceus, Sterculia setigera, Grewia bicolor, Bombax costatum, Adansonia digitata et Acacia albida ;

- Le groupe II: avec FL supérieur ou égal à $80 \%$ et inférieur à $100 \%$ représenté par des espèces comme Acacia senegal, Ziziphus mauritiana, Grewia flavescens, Grewia tenax, Leptadania hastata, Anogeissus leiocarpus, Guiera senegalensis et Calotropis procera;

- Le groupe III : FL compris entre 60 et $80 \%$ regroupant Boscia angustifolia, Cordyla pinnata et Feretia apodanthera;

- Le groupe IV : FL supérieur à $50 \%$ et inférieur à $60 \%$ avec Combretum glutinosum, Sclerocarya birrea et Azadirachta indica ; et

- Le groupe $V$ avec une fidélité égale ou inférieure à $50 \%$ associant des espèces comme Cadaba farinosa, Combretum micranthum, Piliostigma reticulatum, Acacia nilotica, Tamarindus indica, Mitragyna inermis, Pterocarpus lucens et Khaya senegalensis.

Tableau 4 : Importance des ligneux fourragers : niveau de fidélité (FL).

\begin{tabular}{llr}
\hline Fourrage & Usages & FL \\
\hline Pterocarpus erinaceus & Médicaments, Fourrage, Bois & 100 \\
Sterculia setigera & Alimentation & 100 \\
Grewia bicolor & Fourrage, Médicament & 100 \\
Bombax costatum & Fourrage, Service & 100 \\
Adansonia digitata & Fourrage, Alimentation, Médicament & 100 \\
Acacia albida & Fourrage, Médicament & 100 \\
Acacia senegal & Fourrage, Bois, Médicament & 94,11 \\
Ziziphus mauritiana & Alimentation, Fourrage, Médicaments & 94,11 \\
Grewia flavescens & Fourrage, Bois & 94,00 \\
Grewia tenax & Fourrage, Bois & 92,55 \\
Leptadenia hastata & Fourrage, Alimentation & 92,85 \\
Anogessus leiocarpus & Fourrage, Médicament & 88,88 \\
Guiera senegalensis & Médicament, Fourrage, Bois \\
Calotropis procera & Fourrage, Médicament & 84,00 \\
Diospyros mespiliformis & Fourrage, Médicament & 83,33 \\
Boscia angustifolia & Fourrage, Médicament & 80,00 \\
Cordyla pinnata & Fourrage, Alimentation, Médicament & 76,92 \\
Feretia apodanthera & Fourrage, Alimentation & 72,00 \\
Combretum glutinosum & Médicament, Fourrage, Bois & 60,00 \\
Sclerocarya birrea & Alimentation, Fourrage, Bois & 57,89 \\
Azadirachta indica & Fourrage, Bois, Ombrage & 55,00 \\
Cadaba farinosa & Fourrage, Médicament & 52,17 \\
Combretum micranthum & Alimentation, Fourrage, Médicaments & 50,00
\end{tabular}


Piliostigma reticulatum

Acacia nilotica

Tamarindus indica

Mitragyna inermis

Pterocarpus lucens

Khaya senegalensis
Fourrage, Bois

50,00

Fourrage, Bois

40,00

Alimentation, Fourrage

40,00

Fourrage, Bois

Bois, Fourrage

Médicament, Bois, Fourrage
40,00

40,00

40,00
Rôle dans la pharmacopée traditionnelle: Les ligneux font l'objet d'utilisation à des fins médicamenteux pour l'ensemble des populations dont la plupart utilise effectivement une partie particulière de l'arbre pour certains traitements. Ainsi, 27 espèces ligneuses appartenant à diverses familles botaniques sont utilisées pour traiter des cas particuliers de maladies (Voir tableau 5).

Tableau 5 : Les ligneux utilisés dans la pharmacopée traditionnelle et les traitements.

\begin{tabular}{|c|c|c|c|c|}
\hline Espèces & Nb. cit. & $\mathrm{VU}$ & Traitement & $\mathrm{PU}$ \\
\hline Pterocarpus erinaceus & 37 & 0,82 & Fatigue, tension & Ec/Feui \\
\hline Cassia sieberiana & 17 & 0,37 & Parasitoses & $\operatorname{Rac}$ \\
\hline Sclerocarya birrea & 17 & 0,37 & Fatigue & Frts/Ec \\
\hline Balanites aegyptiaca & 17 & 0,37 & Tension/ Fatigue & Racine/ Frts \\
\hline Guiera senegalensis et Combretum & & & & \\
\hline glutinosum & 12 & 0,26 & Rhumes & Feui \\
\hline Combretum micranthum & 11 & 0,24 & Rhumes & Ec \\
\hline Grewia bicolor & 11 & 0,24 & Fatigue & Ec \\
\hline Cordyla pinnata & 11 & 0,24 & Parasitoses & Feui/Ec \\
\hline Boscia angustifolia & 10 & 0,22 & Impuissance & Ec \\
\hline Acacia nilotica & 10 & 0,22 & Douleurs abdominales & Frts \\
\hline Sterculia setigera & 10 & 0,22 & Parasitoses/ Fièvre & Ec \\
\hline Combretum lecardii & 9 & 0,20 & Fatigue & Feui/Frts \\
\hline Tamarindus indica & 9 & 0,20 & Fatigue & $\operatorname{Rac}$ \\
\hline Pavetta oblongifolia & 8 & 0,17 & Dermatose & Feui/Rac \\
\hline Ziziphus mauritiana & 8 & 0,17 & Diarrhées, Maux de tête & $\operatorname{Rac}$ \\
\hline Mangifera indica & 8 & 0,17 & Tétanos & Feui \\
\hline strychnos spinosa & 7 & 0,15 & Impuissance & Rac \\
\hline Anogeissus leiocarpus & 7 & 0,15 & Mystique & Feui/Ec \\
\hline Feretia apodanthera & 5 & 0,11 & Mystique/ Fièvre & Feui \\
\hline Crossopteryx februfuga & 4 & 0,08 & Fatigue & Feui \\
\hline Combretum nigricans & 3 & 0,06 & Fatigue & Ec \\
\hline Heeria insignis & 2 & 0,04 & Impuissance/ Anti venins & Feui/Rac \\
\hline Adansonia digitata & 1 & 0,02 & Diarrhées, Fatigue & Frts \\
\hline Acacia macrostachya & 1 & 0,02 & Fatigue & Racine \\
\hline Azadirachta indica & 1 & 0,02 & Maux de tête & Feui \\
\hline Bombax costatum & 1 & 0,02 & Fièvre & Ec \\
\hline
\end{tabular}

Les enquêtes révèlent aussi que l'arbre constitue essentiellement le meilleur moyen de traitement pour les animaux en cas de maladies. En effet, il est rapporté que l'animal très souvent en cas de maladies, change son habitude alimentaire et cueille même parfois des feuilles qu'il n'utilisait pas en temps normal. Sont ainsi traités chez les humains, la fatigue corporelle, certaines dermatoses, fièvre, les douleurs, rhume, tensions, parasitoses, ou antidote contre les venins de serpents. Toutes les parties de la plante peuvent être souvent utilisées mais il y'en a certaines qui sont réputées comme plus efficaces. Ces parties peuvent être soient les feuilles, les racines, l'écorce ou la gomme. Ces parties peuvent être soit trempées dans de l'eau directement pour être prêtes à l'emploie, ou séchées puis moulues avant d'être utilisées. II est 
rapporté que les feuilles de Guiera senegalensis seules ou en association avec celles de Combretum glutinosum luttent très efficacement contre les états de rhinites. Dans le cas de Pterocarpus erinaceus de valeur usuelle très élevée $(0,82)$, l'espèce est bien appréciée dans le cas de traitements de fatigue générale chez l'Homme tout comme chez les chevaux et ses feuilles comme son écorce, peuvent bien être utilisées pour cela. L'écorce peut simplement être trempée mais les feuilles doivent être bouillies avant utilisation. La racine de Heeria insignis $(\mathrm{VU}=0,04)$ est très fortement recherchée par les locaux à cause des vertus aphrodisiaques que l'on lui prête dans la zone. La famille la plus représentée en terme spécifique est celle des Combretaceae (7 espèces), suivie de celles des Anacardiaceae et Rubiaceae ( 3 espèces chacune) (figure 5).

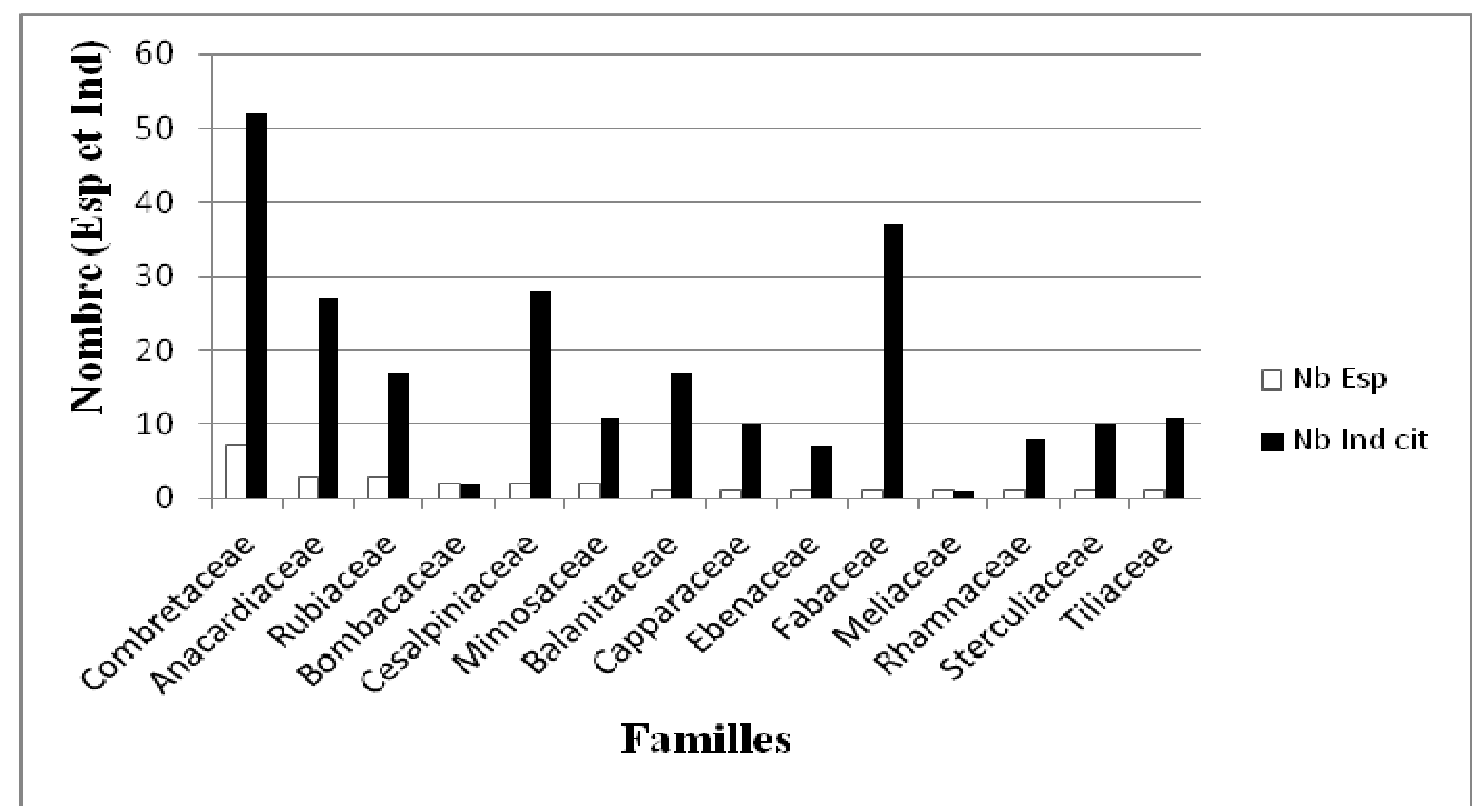

Figure 5 : Distribution des espèces et individus à travers les familles taxonomiques

Rôle dans l'artisanat, l'énergie et la production de bois: L'artisanat sollicite particulièrement presque toutes les parties de l'arbre (écorce, tige, racine, tronc). Le bois d'œuvre et d'énergie (bois de chauffe et production de charbon) constituent des domaines où le bois est particulièrement exploité et souvent de façon non réfléchi. Pour se procurer de l'énergie ou du charbon de bois, les populations utilisent le plus souvent le bois de Combretum glutinosum (VU=0,95) ou celui de Pterocarpus erinaceus (VU= 0,46). Dans le cas de cette dernière, il est rapporté que c'est uniquement les pieds morts que l'on a le droit utiliser pour des besoins d'énergie. Cela contribue au déboisement abusif du couvert ligneux. II faut aussi noter l'importance du cordage du Baobab (Adansonia digitata) très utilisé, surtout pour la confection de cases ou de clôtures et aussi souvent les branches d'Azadirachta indica $(\mathrm{VU}=0,22)$ ou d'Eucalyptus $s p$ (essence reboisée dans la zone de $\mathrm{VU}=0,33$ ), par substitution de Bombax costatum (VU= 0,22) ou Sclerocarya birrea $(\mathrm{VU}=0,20)$ pour les toits de maisons. 
Tableau 6 : Rôle dans la production de bois et dans l'artisanat.

\begin{tabular}{lccc}
\hline Espèces & Nb. cit. & VU & Usage \\
\hline Combretum glutinosum & 43 & 0,95 & Feu-Service \\
Pterocarpus erinaceus & 21 & 0,46 & Feu \\
Cordyla pinnata & 15 & 0,33 & Feu \\
Eucalyptus sp & 15 & 0,33 & Service \\
Guiera senegalensis & 14 & 0,31 & Feu-Service \\
Pterocarpus erinaceus & 11 & 0,24 & Service \\
Cordyla pinnata & 11 & 0,24 & Service \\
Hannoa undulata & 10 & 0,22 & Feu \\
Bombax costatum & 10 & 0,22 & Service \\
Azadirachta indica & 10 & 0,22 & Service \\
Sclerocarya birrea & 9 & 0,20 & Service \\
Anogeissus leiocarpus & 8 & 0,17 & Feu \\
Balanites aegyptiaca & 7 & 0,15 & Service \\
Combretum micranthum & 5 & 0,11 & Feu \\
Acacia macrostachya & 5 & 0,11 & Service \\
Piliostigma reticulatum & 3 & 0,06 & Feu \\
Feretia apodanthera & 3 & 0,06 & Feu \\
\hline
\end{tabular}

$\mathrm{Nb}$. Cit : Nombre de citation; VU : Valeur usuelle ;

A la recherche de liens espèces - services écosystèmiques. : Nous observons ici que le plan factoriel formé par les deux premiers axes (F1 et F2) totalise près de $57 \%$ de l'information. L'analyse peut donc être faite sur ceux-ci (figure 6). L'axe F1 oppose ainsi deux groupes (GI et GII). Le groupe $\mathrm{GI}$ contient les espèces utilisées dans l'alimentation des hommes (Al) avec une contribution de $81,37 \%$ dans l'axe. Elles sont au nombre de 18. Celles qui contribuent le plus à cet axe sont Moringa oleifera (Mool), Terminalia avicenniodes (Teav), Gardenia erubescens (Gaer), Afzelia africana (Afaf), Ziziphus gola (Zigo) et Ficus gnaphalocarpa (Fign) qui sont des espèces à usage alimentaire exclusive. Le groupe GII contient les espèces qui sont à la fois utilisées dans la pharmacopée traditionnelle $(\mathrm{Ph})$, le fourrage $(\mathrm{Fo})$, l'énergie (En) et la construction (Co). II s'agit donc du groupe d'espèces servant à plusieurs usages. Elles sont au nombre de 29. L'axe F2 permet de scinder le groupe GIl en deux sous-groupes (GII1 et GII2). Le sous-groupe GII1 caractérise le service de régulation Pharmacopée (avec 56,52\% de contribution sur l'axe). II contient les espèces Acacia macrostachya (Acma), Pavetta oblogifolia (Paob), Heeria insignis (Hein), Crossopteryx februfuga (Crfe), Cassia sieberiana (Casi), Combretum lecardii (Cole), Combretum nigricans (Coni), Grewia bicolor (Grbi), Sclerocarya birrea (Scbi), Bombax costatum (Boco), Mangifera indica (Main), etc. C'est le sous groupe des espèces qui sont beaucoup plus utilisées dans la pharmacopée. Le sous groupe GII2 représente le service d'approvisionnement Fourrage $(34,17 \%$ de contribution). Ici nous avons les espèces telles que Guiera senegalensis (Guse), Acacia seyal (Acse), Anogeissus leiocarpus (Anle), Pterocarpus lucens (Ptlu), Grewia flavescens (Grfl), Mitragyna inermis (Miin), Grewia tenax (Grte), Khaya senegalensis (Khse), Piliostigma reticulatum (Pire), etc. Ces espèces ont un caractère fourrager prépondérant. 


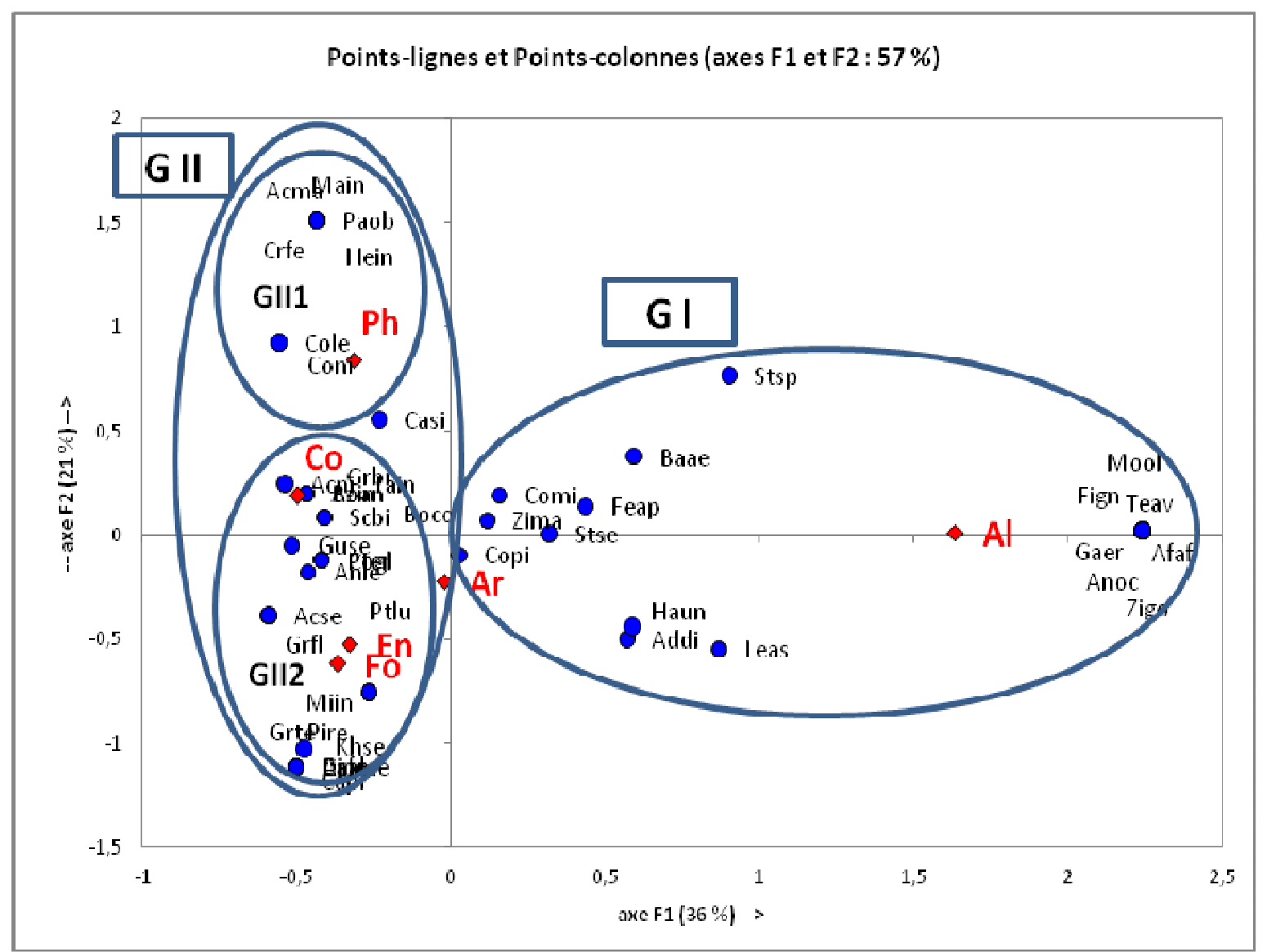

Figure 6 : Projection des individus (espèces) et variables (Usages) sur les plans factoriels F1×F2.

\section{DISCUSSION}

Dans les terroirs villageois de Lour Escal et de Ida Mouride, l'arbre est au centre de toutes les activités des populations. Ainsi, 47 espèces ligneuses contribuent à la fois dans l'alimentation des Hommes et du bétail, la pharmacopée, la production de bois et dans l'artisanat. Ces arbres servent à plusieurs usages, ce sont des arbres agroforestiers (Bonkoungou, 1993).Pour tous ces types d'usages cités, il existe un niveau de consensus élevé (supérieur à $60 \%$ ). L'arbre assure donc à la fois plusieurs fonctions : un tel arbre est donc un arbre à plusieurs usages. C'est ainsi que Pterocarpus erinaceus entre autres, assure à la fois une fonction fourragère ( $\mathrm{VU}=86 \%$ ), une fonction médicamenteuse ( $\mathrm{VU}=82 \%)$ et dans la production de bois $(\mathrm{VU}=0,33)$. Dans la localité, les populations ont conscience du rôle écologique de l'arbre en ce sens qu'il intervient dans le maintien des dunes de sable et la viabilité des mares temporaires. II assure ainsi un rôle de régulation. Cette fonction est notée par plusieurs auteurs. Akpo (1993) note que l'arbre modifie les conditions écologiques en réduisant le pouvoir évaporant de l'air et en favorisant le bilan hydrique. Le rôle écologique des ligneux est donc bien connu des ruraux. C'est d'ailleurs ce que Diouf (2012) a constaté en réalisant des études de perception dans la zone du Ferlo au Nord du Sénégal. Ce dernier montre que près de $67 \%$ de la population du Ferlo a conscience de ce rôle et entretient des activités de reboisement en vue d'améliorer les conditions écologiques (anti-érosion, maintien fertilité).Dans l'alimentation des hommes comme des troupeaux, l'arbre occupe une place centrale. Cette importance est perceptible en observant les valeurs usuelles élevées pour ces deux types d'usages $(64 \%$ et $40 \%)$ respectivement pour le pâturage et l'alimentation pour les humains. Ce qui montre que l'arbre assure une fonction d'approvisionnement à l'homme et à ses animaux d'élevages et est un recours nécessaire en milieu rural. C'est ce même constat qu'a fait Lykke et al (2002) dans le Sahel. , Ganaba et al (2012) notent aussi l'importance de l'arbre dans l'alimentation, en ce sens qu'il constitue un apport nutritionnel si bien que Bekker 
(1983) et Jacks (1994) affirment que les ligneux constituent la principale source alimentaire pour les populations démunies.

Les humains utilisent beaucoup plus les fruits des arbres pour s'approvisionner. En effet, les espèces à grande consommation sont représentées par des espèces fruitières telles que Cordyla pinnata, Adansonia digitata, et Gardenia erubescens avec respectivement des valeurs usuelles de $1 ; 0,77$ et 0,57 . Dans le cas des deux premières citées, elles sont tolérées dans les champs et cohabitent avec les cultures formant ainsi des parcs agroforestiers. D'ailleurs pour Cordyla pinnata, Sall (1996) rapporte que les paysans du Saloum n'ont noté aucune influence négative de cette espèce sur le rendement $d u$ mil et de l'arachide. C'est la raison pour laquelle, en plus de sa fonction, elle est sans doute l'espèce la mieux représentée dans les champs de la localité.

$61 \%$ des espèces citées sont utilisées comme fourrage. Ce qui montre que le rôle fourrager de l'arbre est prépondérant par rapport aux autres types d'usages dans la localité. Cela est du à l'importance de l'élevage transhumant et semi-transhumant dans la zone. Cette importance du caractère fourrager de l'arbre est aussi notée par Lykke et al (2002). Ce dernier, en étudiant 56 espèces ligneuses dans le Sahel, note que $95 \%$ d'entre elles sont des fourrages importants. Cependant, on note une certaine hiérarchisation de ces fourrages ligneux. En effet, les cinq (5) niveaux de fidélité définis le montrent. C'est ainsi que certaines espèces sont présentées comme étant les fourrages préférés des ruraux. On peut citer Pterocarpus erinaceus, Sterculia setigera, Grewia bicolor, Bombax costatum, Adansonia digitata et Acacia albida appartenant au niveau I (Fidélité maximale). Néanmoins, 24 espèces sur les 29 fourragères présentent un niveau de fidélité supérieur ou égal à $50 \%$. Cela traduit globalement que dans la zone, les ligneux fourragers sont considérées par les populations comme étant de qualité. Dans le cas de Khaya senegalensis, son rôle fourrager n'est pas très important pour les usagers, cela se traduit par un faible niveau de fidélité ( $40 \%$ qui est le niveau le plus bas de la liste) et aussi une valeur usuelle très faible (17\%). Ce constat est aussi fait par Lykke et al pour la même

\section{CONCLUSION}

Le présent travail montre la place et la fonction que l'arbre occupe dans le vécu quotidien des agriculteurs et pasteurs dans les parcours des communautés rurales de Lour Escale et de Ida Mouride. Les ligneux assurent des fonctions aussi importantes et diverses et espèce en 2002. Cependant, les valeurs de fidélité faibles pour les des espèces comme Pterocarpus lucens et Acacia nilotica relèveraient plus de leur rareté voire de leur absence, donc de leur méconnaissance dans la zone. Ce qui laisse croire que les populations accordent beaucoup plus d'attention aux espèces les plus abondantes dans la zone. En effet, ces dernières sont très bien connues dans la zone pastorale du Ferlo où Ndiaye et al (2010) rapportent qu'elles sont bien appétées. Cet état de fait peut être confirmé par la faible valeur usuelle de l'espèce Heeria insignis qui est une espèce disparue dans la localité (Sarr, 2009). Outre un service alimentaire et fourrager, l'arbre constitue aussi recours pour les traitements de divers maux dans la localité. C'est d'ailleurs ce qui explique le niveau de fidélité de $89 \%$ pour la médicamentation et aussi la valeur usuelle de $57 \%$ très considérable pour ce même facteur. Cela montre que dans les territoires villageois de ces communautés rurales, les agroéleveurs et pasteurs ont recours systématiquement à l'arbre pour se soigner. Ce qui semble aussi le cas dans le Néma où Ngom et al (2003) rapportent que $67 \%$ des ligneux sont utilisés pour la pharmacopée. C'est ce qui expliquerait d'ailleurs le nombre élevé de tradipraticiens en milieu rural. Ce nombre est évalué dans la zone du Ferlo à environ $11 \%$ par Ndiaye et a (2010). L'analyse des résultats fournis par l'AFC nous permet d'affirmer que $F 1$ indique un gradient d'anthropisation. En effet, les espèces contribuant très fortement à cet axe (caractérisé par la variable Alimentation) sont des espèces exclusivement à usage alimentaire. Quelques unes sont plantées par les populations soit pour leurs feuilles (Moringa oleifera), soit pour leurs fruits (Ziziphus gola et Anacardium occidentale) et sont donc appropriées et surveillées. Cette appropriation de l'arbre dans les terroirs villageois a été notée par Ngom et al (2003). En revanche, pour ce qui est des espèces de la marge des coordonnées négatives de l'axe F1 caractérisant les autres types d'usages (Pharmacopée, Fourrage, Energie, Construction et Artisanat), ce sont surtout des espèces à usages multiples, non protégées et témoignant donc une pression forte sur celles-ci vue leurs différents services offerts.

jouent ainsi un rôle social très important. Ils interviennent dans: l'alimentation du bétail et des Hommes, la pharmacopée, l'artisanat, la production de bois d'énergie et de charbon. Le niveau de Fidélité des ligneux fourragers et le Facteur de consensus 
informateur des types d'usages généralement très élevés montrent que les populations ont une véritable connaissance de l'arbre et lui accordent une importance capitale dans leurs diverses activités. Cependant, les mêmes espèces ligneuses assurent à la fois plusieurs rôles aussi importants les uns des autres : ce sont des ligneux à usages multiples. L'arbre occupe donc une place importante dans la contribution des écosystèmes à la satisfaction des besoins socioéconomiques. Malgré cette multitude de fonction, I'AFC

\section{BIBLIOGRAPHIE}

Adam JG 1970. Noms vernaculaires des plantes du Sénégal. CNRS. J. Agric. Tropic. Bot. Appl., 17, 7-9.

Akpo LE 1993. Influence du couvert ligneux sur la structure et le fonctionnement de la strate herbacée en milieu sahélien. Les déterminants écologiques.TDM, Paris : ORSTOM, 174p.

Akpo LE 1997. Phenological interactions between tree and under story herbaceous vegetation of a sahelian semi-arid savana. Plant Ecology, $131: 241-248$.

Akpo LE et Grouzis M 1996. Influence du couvert sur la régénération de quelques espèces ligneuses sahéliennes (Nord Sénégal, Afrique Occidentale). Webbia, 50 (2), 247-263.

ANAMS (Agence Nationale de Météorologie du Sénégal), données climatologiques (températures et précipitations) des zones de Kaffrine et Koungheul de 1965 à 2008.

Becker B 1983. The contribution of wild plants to human nutrition in the Ferlo (Northern Senegal). Agroforestry Systems, $1: 257-267$

Berhaut J 1967. Flore du Sénégal. BERHAUT J. (ed.), Edition Clair Afrique, Dakar, $485 \mathrm{p}$.

Betti JL 2007. Stratégie/plan d'action pour une meilleure collecte des données statistiques sur les produits forestiers non ligneux au Cameroun et recommandations pour les pays de la COMIFAC, 170p.

Boudet G 1975. Manuel sur les pâturages tropicaux et les plantes fourragères. Ministère de la coopératation, Paris, 4, 254.

Bonkoungou EG, Ayuk AT et Zoungrana I 1993. Les parcs agro-forestiers des zones semi-arides d'Afrique de l'ouest. Symposium international, Wagadougou, Burkina Faso, 226 pages.

Breman H, Ridder N, DE Ketelaars JJMH et Keuken VAN H, 1991. Manuel sur les pâturages des pays sahéliens. Paris, Khartala. indique une liaison espèce-Usages très forte témoignant d'une pression exercée par l'homme sur l'arbre pour satisfaire ses besoins. Dès lors, des moyens de gestion et de réhabilitation des écosystèmes doivent être analysés avec les populations afin de permettre aux décideurs publics et privés d'avoir des outils pratiques pour une exploitation rationnelle de la ressource dans les parcours communautaires afin d'assurer leur durabilité.

Canales M, Hermandes T, Caballero J, Romo DE Vivar A, Avila G, Duran A et Lira R, 2005. Informant consensus factor and antibacteriel activity of the medicinal plants used by the people of San Rafael coxcatlan, Puebla, Mexico, Journal of Ethnopharmacology, 97 : 429-439.

Cheikh youssef A, Ashekele HMU, Shapi M et Matengu $\mathrm{K}, 2011$. Ethnobotanical study of indigenus knowledge on medicinal plant use by traditional healers in Oshikoto region, Namibia, Journal of Ethnobiology and Ethnomedicine, $7: 10$

Diouf JC 2012. Dynamique du peuplement ligneux au Ferlo (Nord-Sénégal), conséquences et perspectives pour une gestion durable, Thèse, UCAD-FST.

FAO 1992. Manuel sur la foresterie rurale. Tome1: zone du bassin arachidier/Projet d'appui au programme national de foresterie rurale, $328 \mathrm{p}$.

Ganaba S, Ouadba J, et Bognounou O, 2002. Utilisation des ressources végétales spontanées comme aliments complémentaire en région sahélienne du Burkina Faso. Annales de Botanique de l'Afrique de l'Ouest, 2: 101-1011.

Giffard PL, 1974. L'arbre dans le paysage sénégalais. Sylviculture en zone tropical sèche, CTFT, Dakar, 431p.

Jacks B, 1994. Living conditions and nutrition of some Tamasheq groups in Mali under the influence of drought, Ambio 23: 438-445.

Lebrun JP, Stork AL, 1991, 1992, 1995, 1997. Enumération des plantes à fleurs d'Afrique tropicale. Conservation du jardin botanique de Genève, LEBRUN J.P. et STORK A.L. (eds), Volumes 1-2-3-4, pp.: 1-2-3-4, pp.: 249, pp.: 257, pp.: 341 et 712 . 
Le Houerou HN, 1980. Browse in Africa: The current state of knowledge.Adis Abéba. Internationale livestock Center for Africa.

Lykke AM, Merzt $O$ et Ganaba S, 2002. Food consumption in rural Burkina Faso. Ecology of Food and Nutrition, 41 : 119-153.

Ndiaye M, Akpo LE, Dione ME, 2010. Caractéristiques des ligneux fourragers dans les terroirs pastoraux de RANEROU (Région de Matam, Nord-Sénégal), Journal. Sci. 10, №3:12-27.

Ngom D, Banoin M, Dacosta H et Akpo LE, 2003. Perception de l'importance de l'arbre en pays Sérer: Le cas du bassin de la Néma en zone soudano-sahélienne du Sénégal, Etude et recherches sahélienne $N^{\circ} 10$.

Sarr 0 2009. Caractéristiques des ligneux fourragers dans les parcours communautaires de Lour Escale (région de Kaffrine centre-Senegal), 53p.

Sall PN 1996. Les parcs agroforestiers au Sénégal: Etats des connaissances et perspectives.

Touré A 2002. Contribution à l'étude de l'évolution des réservoirs de carbone en zone nord soudanien au Sénégal. Doctorat en Sciences biologiques, EPFL, Lausanne, 220p.

Von Maydell HJ 1990. Trees and shrubs of the Sahel: Their characteritics and Uses, GTZ, 531p. 